\title{
A Robust Star Identification Algorithm with Multi- resolution Radial Pattern and Its Hardware Implementation
}

\author{
Qiang Wu, Zuwu Zhu, Xin Zheng \\ Department of Information \\ Beijing University of Technology \\ Beijing, China \\ wuqiang@bjut.edu.cn
}

\begin{abstract}
Star sensor is a device that uses astronomical information for navigation and attitude measurement. It is widely used in satellites, intercontinental missiles, strategic bombers and other scenarios. Since star sensors are mainly used in the military field, we need a fast, robust and high accuracy star identification algorithm. In order to improve the performance of the star sensor, this paper will use the Haar wavelet transform to obtain the multi-resolution radial features of the star image, which is matched according to the resolution from low to high. The algorithm is implemented in a pipelined manner on the FPGA platform. Experiments show that the star identification rate of the scheme reaches $98 \%$ or more when the star position error is within 3 pixels, and the worst case time consuming is only 1.4 millisecond when the FPGA runs at $100 \mathrm{MHZ}$. Compared with the traditional algorithm, this is a more robust and real-time star identification algorithm.
\end{abstract}

Keywords—Star Sensor, Radial Pattern, FPGA,

\section{INTRODUCTION}

Star sensor is a device that uses astronomical information for navigation and attitude measurement. It is widely used in satellites, warship, airplane and other scenarios[1].The work of the star sensor can be roughly divided into three parts[2]: 1. Extracting the position of the star in a star image; 2.star identification; 3. Calculating the posture. Since star sensors are mainly used in the military field, the accuracy of star identification is not only high, but also fast, and robust. Therefore, it is necessary to research a robust, real-time, and high identification rate star identification algorithm.

With the development of star sensors, more and more star identification algorithms have been proposed. In general, the star identification algorithm can be divided into subgraph isomorphism and pattern recognition. Junkins[3] first proposed the use of triangles for star identification, which can be considered as the beginning of the subgraph isomorphic star identification algorithm. Later, Groth and Anderson[4] improved the arrangement of the navigation star catalog and improved the search method. In the 1990s, Liebe[5] first completed the celestial autonomous star identification using a triangle algorithm in the star sensor. Lei du[6] improved the identification rate and the recognition time consuming was reduced by $70 \%$ compared with the traditional method. Representative star identification algorithms in the pattern recognition class include grid method[7], polar coordinate transformation[8] method and the like. Zhu Hailong[9] proposed the OSP pattern feature for star identification with the k-vector proximity method. Yang Zhao[3] improved radial featurebased algorithms have achieved good results. In addition, neural network-based star identification algorithms[10] are also devolving. The research work in this paper is based on the previous studies.

Most of the current star identification algorithms are based on DSP/ARM architecture. It is difficult to perform parallel computing, and real-time performance is difficult to guarantee. With the development of FPGAs, computing and storage capabilities are becoming more and more powerful. Ordinary FPGAs can store the entire star catalog, which makes it possible to perform star identification on the FPGA. The star identification algorithm based on the FPGA platform is a true parallel computing and meet the real-time requirements of the star sensor.

The radial pattern has rotation invariance and is simple in construction, suitable for hardware parallel computing, but the identification rate is greatly affected by the quantization level. In this paper, the Haar wavelet transform is used to obtain radial pattern of multiple resolutions, and the matching is performed in order of resolution from low to high, thereby improving the identification rate. The entire algorithm will be calculated in pipeline to improve the speed of star identification.

\section{TRADITIONAL RADIAL PATTERN}

\section{A. Angular distance calculation}

In star identification, angular distance is generally used to characterize the distance between star points. We assume that $s_{i}\left(\alpha_{i}, \delta_{i}\right)$ and $s_{j}\left(\alpha_{j}, \delta_{j}\right)$ are two stars on the celestial coordinate system, $\alpha$ for the right ascension and $\delta$ for the declination. The position of $s_{i}$ and $S_{j}$ in the celestial Cartesian coordinate system can be expressed as Equation (1). 


$$
v_{i}=\left[\begin{array}{c}
\cos \alpha_{i} \cos \delta_{i} \\
\sin \alpha_{i} \cos \delta_{i} \\
\sin \delta_{i}
\end{array}\right], \quad v_{j}=\left[\begin{array}{c}
\cos \alpha_{j} \cos \delta_{j} \\
\sin \alpha_{j} \cos \delta_{j} \\
\sin \delta_{j}
\end{array}\right]
$$

Assuming $p_{i}$ and $p_{j}$ is the star points on the star image, $\left(x_{i}, y_{i}\right)$ and $\left(x_{j}, y_{j}\right)$ as the centroid coordinates of the star point. The unit vector of $p_{i}$ and $p_{j}$ under the star sensor coordinate system can be expressed as Equation (2), where $f$ is the focal length of the star sensor.

$w_{i}=\frac{1}{\sqrt{x_{i}^{2}+y_{i}^{2}+f^{2}}}\left[\begin{array}{c}x_{i} \\ y_{i} \\ -f\end{array}\right], \quad w_{j}=\frac{1}{\sqrt{x_{j}^{2}+y_{j}^{2}+f^{2}}}\left[\begin{array}{c}x_{j} \\ y_{j} \\ -f\end{array}\right]$

The angular distance between $S_{i}, s_{j}$ in the celestial Cartesian coordinate system and $p_{i}, p_{j}$ in the star sensor coordinate system are expressed as Equation (3).

$d_{i, j}^{c}=\arccos \left(\frac{v_{i} \cdot v_{j}}{\left|v_{i}\right| \cdot\left|v_{j}\right|}\right) \quad, \quad d_{i, j}^{s}=\arccos \left(\frac{w_{i} \cdot w_{j}}{\left|w_{i}\right| \cdot\left|w_{j}\right|}\right)$

According to the projection principle, in the ideal case, $d_{i, j}^{c}$ should be equal to $d_{i, j}^{s}$.Therefore, it is possible to use a corner distance as a basic element to construct a suitable star pattern for star identification.

\section{B. The Generation of radial pattern}

For the convenience of representation, the angular and radial pattern calculated by using the data in the navigation star table are respectively represented by $d_{i, j}^{c}$ and cpat ; the angular and radial pattern calculated by the star point information obtained from the star image are respectively represented by $d_{i, j}^{s}$ and spat $d_{i, j}$ and pat represent angular and radial features that are compatible with both of the above.

A schematic diagram of radial pattern generation is shown in Fig. 1. Assuming the main star is S, PR is the star pattern radius, and $\mathrm{Rd}$ is the minimum radius set to distinguish the double stars. The star mode vector of the main star is determined by other companion star in the PR neighborhood.

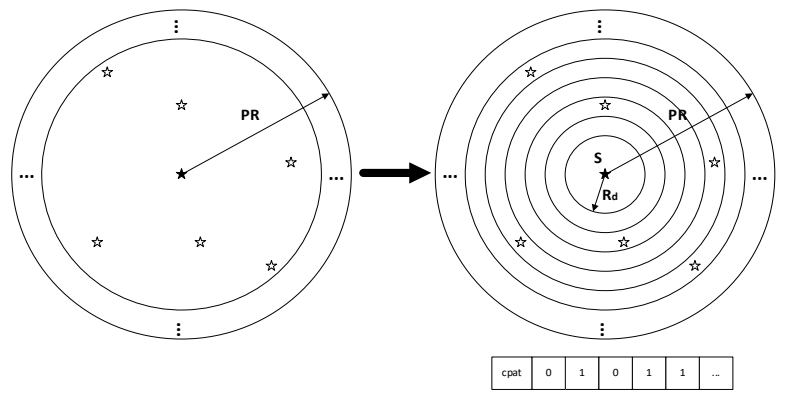

Figure 1 Radial pattern generation
Assuming there is $\mathrm{M}$ companion stars in the main star $\mathrm{S}$ field, denoted as $\left\{s_{1}, s_{2}, \ldots s_{m} \ldots, s_{M}\right\}$. Their angular distance from the main star $\mathrm{S}$ is expressed as

$$
D_{s}=\left\{d_{s, s_{1}}, d_{s, s_{2}}, \ldots d_{s, s_{m}} \ldots, d_{s, s_{M}}\right\} \text {. The quantization level }
$$

is $N_{q}$, then the quantization interval $Q$ is expressed as Equation (4).

$$
Q=\frac{P R-R d}{N_{q}}
$$

The quantization level $N_{q}$ is equal to the dimension $N$ of the star mode vector pat , and pat can be expressed as Equation (5).

$$
\text { pat }_{n}=\left(a_{1}, a_{2}, \ldots, a_{N}\right) \quad, \quad \text { pat }_{n}= \begin{cases}1 & n=\left\lceil\frac{d_{s, s_{m}}}{Q}\right\rceil \\ 0 & \text { Other }\end{cases}
$$

The \lceil\rceil symbol in the formula indicates rounding up. The vectors in the star schema table can be unequal for each other by setting the appropriate quantization level and the star mode radius $\mathrm{PR}$.

Assume that after screening, there are $\mathrm{T}$ navigation stars whose star mode vector is cpat $_{n}^{t}, 1 \leq t \leq T$. The star pattern vector calculated by the star sensor is $s p a t_{n}$, and the matching score expression is shown in Equation (6).

$$
\operatorname{score}(t)=\sum_{n=1}^{N}\left(\text { cpat }_{n}^{t} \& \text { spat }_{n}\right)
$$

If the star pattern with the highest score is greater than a certain threshold, the star corresponding to the star pattern can be considered as a successfully matched star.

\section{Multi-Resolution RAdial PATtern}

With the Haar wavelet transform, radial pattern of multiple resolutions can be obtained. Fig $2 \mathrm{~s}$ a multi-level wavelet transform with radial pattern.

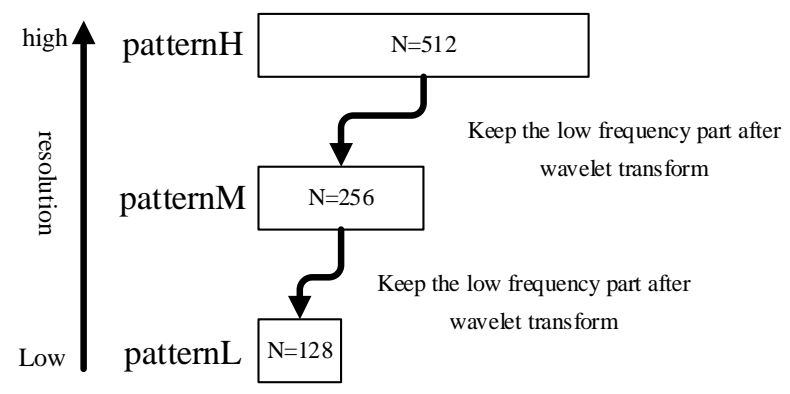

Figure 2 Radial pattern multi-level wavelet transform

In Fig. 2, patternH is the original feature vector with the 
highest resolution; patternM is the low-frequency part of patternH for haar wavelet transform; patternL is the lowfrequency part of patternM for haar wavelet transform. It should be noted that patternH, patternM, and patternL are all binarization features, that is, the components of these feature equal to zero are still zero, and the components that are not zero are all 1.

In the case of matching, the matching score is calculated using Equation (7), and $\oplus$ represents the not xor operation.

$$
\operatorname{score}(t)=\sum_{n=1}^{N}\left(\text { cpat }_{n}^{t} \oplus \text { spat }_{n}\right)
$$

The single matching process is presented.

1) Calculate the matching score of patternL, select the pattern with the highest score and greater than ThresholdL . These patterns is called SETL .

2) If SETL is empty, the match fails. If there is a unique element, the star corresponding to the element is output, and the match ends.

3) If there are multiple elements in SETL, calculate the patternM matching score of these elements, select the star pattern with the highest score and greater than ThresholdM . These patterns is called SETM .
4) If SETM is empty, output the star corresponding to all elements in SETL. If SETM has a unique element, the star corresponding to the element is output, and the matching ends.

5) If the SETM has multiple elements, calculate the patternH matching score of these elements, select the star pattern with the highest score and greater than ThresholdH . These patterns is called SETH .

6) If SETH is empty, output the star corresponding to all elements in SETM. If SETH is not empty, the star points corresponding to these elements are output, and the matching ends.

After this process is finish, if the match fails, we can select other star near the optical axis of the star sensor as the main star for star identification until the match is successful or all the star points are used as main star.

\section{FPGA IMPLEMENTATION}

The method proposed in this paper is implemented in a pipelined manner on the FPGA platform of the model xc7k325tffg676 introduced by Xilinx. As shown in Figure 3, it is the overall design block diagram of the algorithm.

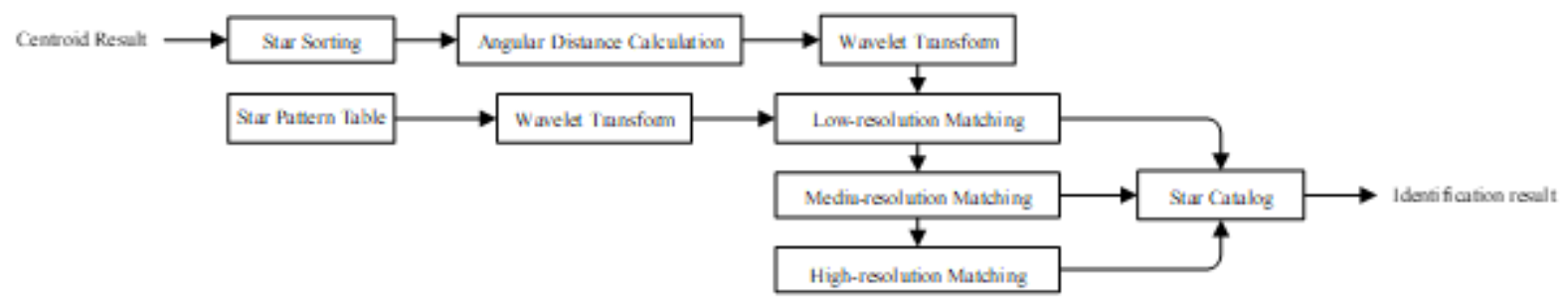

Figure 3 Overall design block diagram

In the Star Sorting module, the distance from the star point to the optical axis of the star sensor is ordered from near to far. The purpose of this step is to select the most suitable main star. This will minimize the loss of border stars. In the Angular Distance Calculation module, the angular distance between the main star and the companion star is calculated and the traditional radial pattern are generated. Since the traditional radial pattern represents the highest resolution, the quantization level can be slightly larger. In the Wavelet Transform module, a Haar wavelet transform that binarizes radial pattern is used to produce radial features of multiple resolutions. The Block RAM is used in the Star Pattern Table module to store radial pattern. The three modules of Low-resolution Matching, Mediumresolution Matching and High-resolution Matching are responsible for implementing the above matching strategy, and then output the matched star point number to the Star Catalog module, which stores the position of the navigation star. Finally, the output of the right ascension declination matching the star point completes the star identification.

When the algorithm is implemented on an FPGA platform, the operating frequency is set to $100 \mathrm{MHZ}$. The time for a single match is $0.14 \mathrm{~ms}$, and the worst match time is $1.4 \mathrm{~ms}$. The navigation star table occupies about $28 \mathrm{~KB}$ of memory, and the star feature star table occupies about $225 \mathrm{~KB}$ of memory. The algorithm consumes a total of $261 \mathrm{~KB}$ of block RAM resources, that is, 58 pieces of $36 \mathrm{~Kb}$ block RAM, accounting for $13 \%(58 / 445)$ of the total resources. Consumes 1,843 Slices resources, accounting for $3.61 \%$ of the total resources (1843/50950).

\section{EXPERIMENT AND ANALYSIS}

In this paper, the identification rate and the robustness of the algorithm are evaluated by the identification rate under different star position errors and the star identification rate under different false star numbers. It is compared with the traditional star identification algorithm based on radial pattern and the triangle algorithm. The original image was randomly generated by an electronic star simulator, and then the algorithm was tested by adding 1 to 3 false stars or 0 to 3 pixel position errors to a total of 11,000 star images.

The SAO J2000 star catalog is used as the original star catalogue, with the limit star $+6 \mathrm{Mv}$ as the limit. After removing the double star and the variable star, there are 3,610 navigation stars. The parameters of the algorithm are set as follows: the star sensor has an angle of view of $14^{\circ}$.the star mode radius is $\mathrm{PR}=7^{\circ}$. The highest quantization 
level is 512; the image resolution is $1024 * 1024$. ThresholdL is 120 , ThresholdM is 240, and ThresholdH is 480 . In the traditional radial feature-based star identification, the quantization level is set to 256. The test results are shown in Figure 4.

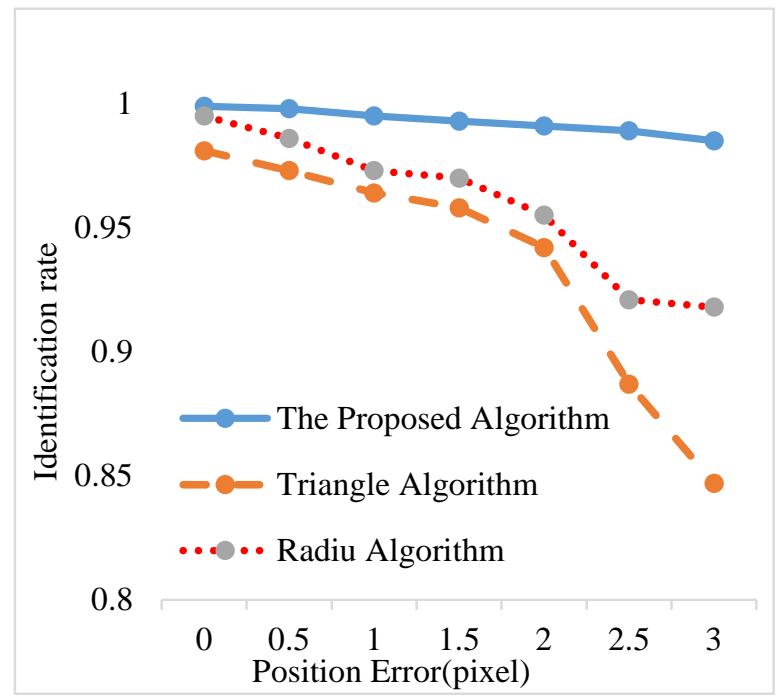

Figure 4a Identification rate versus position error

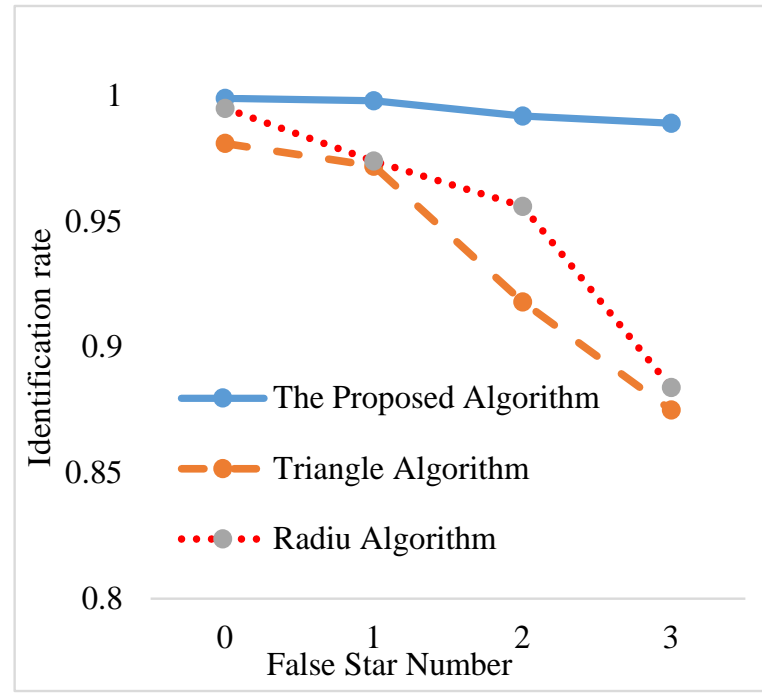

Fig 4b Identification rate versus position error

In Figure 4a, the identification rates of the three algorithms decrease with the increase of the position error of the star point. When the position error is one pixel, the identification rates of the proposed algorithm, radial pattern method and triangle method are $99.5 \%, 97.3 \%$ and $96.4 \%$, respectively. When the position error reaches 3 pixels, the algorithm can still reach The identification rate of $98.5 \%$, while the identification rate of the other two algorithms decreased to $91.8 \%$ and $84.7 \%$, respectively.

Figure 4b shows the anti-jamming capability of the three algorithms for false stars. With the increase of the number of false stars, the identification rate of the radial pattern method and the triangle method decreases rapidly, and the algorithm proposed in this paper still maintains a high level. When there is one false star in the star map, the identification rates of the three algorithms are 99.8\%, 97.4\% and $97.2 \%$, respectively. When there are three false stars in the figure, their identification rates are $98.9 \%, 88.4 \%$ and $87.5 \%$, respectively.

\section{CONCLUSION}

This paper proposes a robust and real-time star identification algorithm. Based on the traditional radial pattern, the Haar wavelet transform is used to construct multi-resolution radial pattern, which are matched in order from low to high resolution. The star point closer to the optical axis of the star sensor is used as the preferred star to improve the identification rate. And the algorithm is implemented in a pipelined manner on the FPGA platform to achieve real-time requirements. Experiments show that the star identification rate of this scheme reaches $98 \%$ or more when the position error of the star point is within 3 pixels and the time consuming in the worst case is only $1.4 \mathrm{~ms}$ Compared with the traditional algorithm, this is a more robust and real-time star identification algorithm.

\section{REFERENCES}

[1] Meng N, Jia P. A survey of all-sky autonomous star identification algorithms, 2006[C].

[2] Yang Z, Wei X, Wang G, et al. Real-time star identification using synthetic radial pattern and its hardware implementation[J]. Acta Astronautica, 2017,131:1-9.

[3] Junkins J L, Iii C C W, Turner J D. Star pattern recognition for real time attitude determination[J]. Journal of the Astronautical Sciences, 1977,25(25):251-270.

[4] Anderson D S. Autonomous star sensing and pattern recognition for spacecraft attitude determination[J]. 1991.

[5] Liebe C C. Pattern Recognition of Star Constellations for Spacecraft Applications[J]. IEEE Aerospace \& Electronic Systems Magazine, 2002,8(1):31-39.

[6] Lei D, Yan Z. An improved triangle star pattern recognition algorithm with high identification probability[J]. Proceedings of SPIE - The International Society for Optical Engineering, 2011,8193(4):819305.

[7] Padgett C, Kreutz-Delgado K. A grid algorithm for autonomous star identification[J]. IEEE Transactions on Aerospace \& Electronic Systems, 1997,33(1):202-213.

[8] Luo L, Xu L, Zhang H, et al. Improved autonomous star identification algorithm[J]. Chinese Physics B, 2015,24(6):321-327.

[9] Zhu H, Liang B, Zhang T. A robust and fast star identification algorithm based on an ordered set of points pattern[J]. Acta Astronautica, 2018.

[10] Tao M A, Sun H H, Xiao S, et al. Recognition algorithm for star pattern of little swatch based on BP neural network[J]. Journal of Applied Optics,2009,30(2):252-256 